\title{
INJECTIVES IN SOME SMALL VARIETIES OF OCKHAM ALGEBRAS
}

\author{
by $\mathrm{R}$. BEAZER
}

(Received 16 February, 1983)

1. Introduction. The study of bounded distributive lattices endowed with an additional dual homomorphic operation began with a paper by $J$. Berman [3]. On the one hand, this class of algebras simultaneously abstracts de Morgan algebras and Stone algebras while, on the other hand, it has relevance to propositional logics lacking both the paradoxes of material implication and the law of double negation. Subsequently, these algebras were baptized distributive Ockham lattices and an order-topological duality theory for them was developed by A. Urquhart [13]. In an elegant paper [9], M. S. Goldberg extended this theory and, amongst other things, described the free algebras and the injective algebras in those subvarieties of the variety $\mathbf{0}$ of distributive Ockham algebras which are generated by a single finite subdirectly irreducible algebra. Recently, T. S. Blyth and J. C. Varlet [4] explicitly described the subdirectly irreducible algebras in a small subvariety MS of $\mathbf{0}$ while in [2] the order-topological results of Goldberg were applied to accomplish the same objective for a subvariety $\mathscr{K}_{1,1}$ of $\mathbf{0}$ which properly contains MS. The aim, here, is to describe explicitly the injective algebras in each of the subvarieties of the variety MS. The first step is to draw the Hasse diagram of the lattice $\Lambda$ MS of subvarieties of MS. Next, the results of Goldberg are applied to describe the injectives in each of the join irreducible members of $\Lambda \mathbf{M S}$. Finally, this information, in conjunction with universal algebraic results due to B. Davey and $\mathrm{H}$. Werner [8], is applied to give an explicit description of the injectives in each of the join reducible members of $\Lambda$ MS.

2. Preliminaries. A distributive Ockham algebra is an algebra $\left\langle L, \vee, \wedge,{ }^{0}, 0,1\right\rangle$ of type $\langle 2,2,1,0,0\rangle$ such that $\langle L, \vee, \wedge, 0,1\rangle$ is a bounded distributive lattice and ${ }^{0}$ is a unary operation defined on $L$ such that, for all $x, y \in L$,

$$
(x \wedge y)^{0}=x^{0} \vee y^{0}, \quad(x \vee y)^{0}=x^{0} \wedge y^{0}, \quad 0^{0}=1, \quad 1^{0}=0 .
$$

The class of all distributive Ockham algebras is a variety, henceforth denoted by $\mathbf{0}$. The subvariety of $\mathbf{0}$ defined by the identity $x \wedge x^{00}=x$ is denoted by $\mathbf{M S}$ and its members are called MS-algebras. The subvariety of MS defined by the identity $x=x^{00}$ is denoted by $\mathbf{M}$ and its members are de Morgan algebras.

Let $\mathbf{K}$ be a class of (similar) algebras. An algebra $I \in \mathbf{K}$ is said to be (weak) injective in $\mathbf{K}$ if, for any algebra $A \in \mathbf{K}$, any (onto) homomorphism from any subalgebra of $A$ to $I$ can be extended to a homomorphism from $A$ to $I . \mathbf{K}$ is said to have enough injectives if each of its members can be embedded into an injective in $\mathbf{K}$. As usual, $\mathbf{I}(\mathbf{K}), \mathbf{H}(\mathbf{K}), \mathbf{S}(\mathbf{K})$ and $\mathbf{P}(\mathbf{K})$ will denote the classes of isomorphic copies, homomorphic images, subalgebras and products of the members of $\mathbf{K}$, respectively. If $\mathbf{K}$ and $\mathbf{M}$ are classes of algebras (both of the

Glasgow Math. J. 25 (1984) 183-191. 
same type) having the property that each member of $\mathbf{K}$ is isomorphic to a member of $\mathbf{M}$ and visa-versa, we shall sometimes abuse conventional notation and write $\mathbf{K}=\mathbf{M}$. In particular, we will always identify $\mathbf{K}$ with $\mathbf{I}(\mathbf{K})$. For all other unexplained lattice theoretic and universal algebraic terminology and notation we refer the reader to [1] or [10]. We also assume basic familarity with the Stone-Priestley duality for bounded distributive lattices, as developed in [11] and [12], at least in the finite case. For our present needs, it will be enough to refer to the fragment of the duality for the class of finite distributive Ockham algebras presented in [2], although for the entire picture we refer the reader to [9] and [13]. $O(X)$ will denote the dual algebra of an Ockham space $(X ; g)$ and $\mathscr{P}(A)$ will denote the dual space of an Ockham algebra $A$. For integers $m>n \geqslant 0, \mathbf{m}_{n}$ will denote the Ockham space $\left(\mathbf{m}, \gamma_{n}\right)$ consisting of the discretely ordered set $\mathbf{m}=\{0,1, \ldots, m-1\}$ and the mapping $\gamma_{n}: \mathbf{m} \rightarrow \mathbf{m}$ defined by

and

$$
\gamma_{n}(k)=k+1 \text {, whenever } 0 \leqslant k<m-1
$$

$$
\gamma_{n}(m-1)=n
$$

The Ockham space $\mathbf{m}_{n}$ may be visualized as in Fig. 1. In [9], M. S. Goldberg shows that any order on $\mathbf{m}_{n}$ with respect to which $\gamma_{n}$ is order reversing gives rise to the dual space of a subdirectly irreducible distributive Ockham algebra and, conversely, all dual spaces of finite subdirectly irreducible distributive Ockham algebras arise in this manner. Therefore, Figure 1 is typical of the diagram of the dual space $X$ of a finite subdirectly irreducible distributive Ockham algebra in which the order relation has been suppressed. The pair consisting of the subposet $L=\{n, n+1, \ldots, m-1\}$ together with the restriction of $\gamma_{n}$ to $L$ is called the loop of $X$.

3. The lattice of subvarieties of MS. We begin with the following key result of B. Davey [7] which is based on consequences of Jónsson's lemma and the Stone-Priestley duality.

THEOREM 1. Let $\mathbf{K}$ be a congruence distributive variety generated by a finite set of finite algebras and let $\mathbf{S i}(\mathbf{K})$ denote the set consisting of precisely one algebra from each of the isomorphism classes of the subdirectly irreducible algebras in $\mathbf{K}$. Then the relation $\leq$ defined

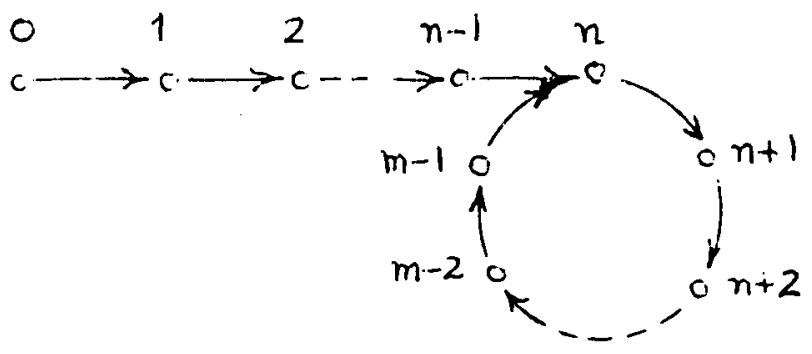

Figure 1 
on $\mathbf{S i}(\mathbf{K})$ by

$$
A \leqslant B \Leftrightarrow A \in \mathbf{H S}(\{B\})
$$

is a partial ordering and the lattice $0(\mathbf{S i}(\mathbf{K}))$ of order ideals of $\langle\mathbf{S i}(\mathbf{K}) ; \leqslant\rangle$ is isomorphic to the lattice $\Lambda \mathbf{K}$ of subvarieties of $\mathbf{K}$ in such a way that the principal order ideal of $\langle\mathbf{S i}(\mathbf{K}) ; \leqslant\rangle$ generated by $A \in \mathbf{S i}(\mathbf{K})$ corresponds to the subvariety $A$ of $\mathbf{K}$ generated by $A$.

The above theorem will be applied in conjunction with the description of the subdirectly irreducible MS-algebras, due to Blyth and Varlet [4], to draw the Hasse diagram of $\Lambda$ MS. In the notation of [2] and [4], there are nine non-isomorphic subdirectly irreducible algebras in MS: $T, B, S, K, K_{1}, K_{2}, K_{3}, M, M_{1}$.

The following simple observation simplifies the construction of $\langle\mathbf{S i}(\mathbf{M S}) ; \leqslant\rangle$ and is crucial to our subsequent discussion of injectivity.

Lemma 2. If $X \in \mathbf{S i}(\mathbf{M S})$ and $\mathbf{X}$ is the subvariety of $\mathbf{M S}$ generated by $X$, then $\mathbf{S i}(\mathbf{X})=\mathbf{S}(\{X\})=\mathbf{H S}(\{X\})$.

Proof. First, observe that $\mathbf{S i}(X) \subseteq \mathbf{H S}(\{X\})$ is a well known consequence of Jónsson's lemma. Let $Z$ be a homomorphic image of a subalgebra $Y$ of $X \in \mathbf{S i}(\mathbf{M S})$. By inspection of the Hasse diagrams in [2] or [4], $Y$ is subdirectly irreducible and so $\mathbf{S}(\{X\}) \subseteq \mathbf{S i}(\mathbf{X})$. Using the results and notation in [2] or [4], we have $Z \cong Y / \theta$, for some congruence $\theta \in\{\omega, \Phi, \iota\}$, so that $Z$ must be isomorphic to $T, Y$ or $Y^{00}$ and therefore $Z \in \mathbf{S}(\{Y\}) \subseteq \mathbf{S}(\{X\})$. Thus, $\mathbf{H S}(\{X\}) \subseteq \mathbf{S}(\{X\})$ and the proof is complete.

Obviously MS is congruence distributive, finitely generated by $M_{1}$ and, in view of Lemma 2, the partial order $\leqslant$ on $\mathbf{S i}(\mathbf{M S})$ is given by

$$
A \leqslant B \Leftrightarrow \mathbf{S}(\{B\}) \text {. }
$$

It is now straightforward to verify that Figs 2 and 3 are Hasse diagrams of $\langle\mathbf{S i}(\mathbf{M S}) ; \leqslant\rangle$ and $\Lambda \mathbf{M S}$, respectively.

Remark. The Hasse diagram for $\Lambda \mathbf{M S}$ along with equational bases for each of its members have been obtained independently by T. S. Blyth and J. C. Varlet [5].

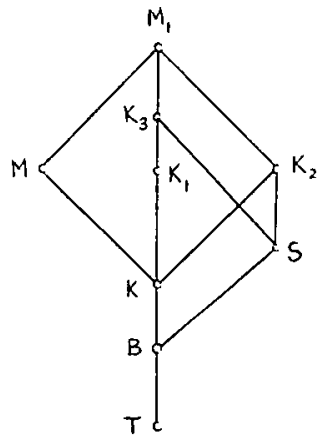

Figure 2 


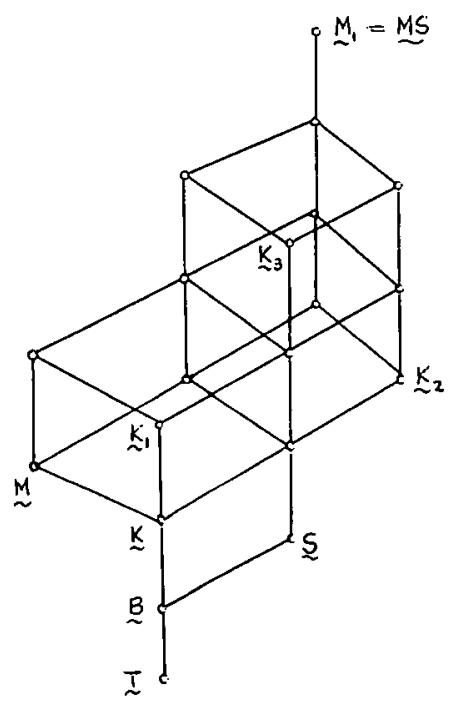

Figure 3

4. Injectives in the subvarieties of $\Lambda$ MS. We begin by alluding to a fundamental universal algebraic construction. Let $B$ be a Boolean algebra whose dual space is $B^{*}$ and let $A$ be an arbitrary algebra. Then the Boolean power of $A$ by $B$, denoted $A[B]$, is the subalgebra of the direct power $A^{B^{*}}$ consisting of all continuous functions from $B^{*}$ into $A$ endowed with the discrete topology. Two elementary properties of this construction which will be needed are: $A[2] \cong A, A\left[B_{0}\right] \times A\left[B_{1}\right] \cong A\left[B_{0} \times B_{1}\right]$. For these and other properties we refer the reader to [6].

In [9], M. S. Goldberg completely described the structure of the injectives in any subvariety of 0 generated by a single finite subdirectly irreducible algebra in 0 in terms of products of Boolean powers of certain algebras in the subvariety by complete Boolean algebras. That part of his theorem which is of particular relevance in the present context may be stated as follows:

Theorem 3. Let $X$ be a finite subdirectly irreducible algebra in $\mathbf{0}$ and let $\mathbf{X}$ be the subvariety of 0 generated by $X$. If $\mathbf{S i}(\mathbf{X})=\mathbf{S}(\{X\})$, then $A \in \mathbf{X}$ is injective in $\mathbf{X}$ if and only if there are complete Boolean algebras $B_{0}$ and $B_{1}$ such that

$$
A \cong X\left[B_{0}\right] \times 0\left(L_{X}\right)\left[B_{1}\right],
$$

where $L_{X}$ is the loop of the dual space of $X$.

Note that, since $X[\mathbf{1}] \cong T$ (the trivial algebra) and $X[\mathbf{2}] \cong X$, both $X$ and $0\left(L_{X}\right)$ are injective in $\mathbf{X}$. Since we observed in Lemma 2 that $\mathbf{S i}(\mathbf{X})=\mathbf{S}(\{X\})$ whenever $X \in \mathbf{S i}(\mathbf{M S})$, it is now purely routine to characterize the injectives in any subvariety $\mathbf{X}$ of $\mathbf{M S}$ generated 
TABLE 1

\begin{tabular}{|c|c|c|c|}
\hline$x$ & $\mathscr{P}(X)$ & $L_{x}$ & $0\left(L_{\mathbf{X}}\right)$ \\
\hline B & & & $B$ \\
\hline$s$ & & & $\boldsymbol{B}$ \\
\hline$K$ & & & $K$ \\
\hline $\boldsymbol{K}_{1}$ & & & $K$ \\
\hline$K_{2}$ & & & $\boldsymbol{K}$ \\
\hline$K_{3}$ & & & $\boldsymbol{K}$ \\
\hline$M$ & & & $M$ \\
\hline$M_{1}$ & & & $M$ \\
\hline
\end{tabular}

by a single subdirectly irreducible algebra $X \in \mathbf{M S}$; that is, in any join irreducible member of $\Lambda$ MS. We tabulate our calculations in Table 1.

Remark. At this point, it is worthwhile pointing out that if $B_{0}$ is a Boolean algebra then $B\left[B_{0}\right] \cong B_{0}$ and $S\left[B_{0}\right] \cong B_{0} * S$, where $B_{0} * S$ is the free product of $B_{0}$ and $S$ in the category of bounded distributive lattices (see, for example, [1], Chapter 7). 
There still remains the problem of characterizing the injectives in the join reducible members of $\Lambda$ MS. By inspection of Fig. 3 there are eleven of them, namely $\mathbf{K} \vee \mathbf{S}, \mathbf{K}_{1} \vee \mathbf{S}$, $\mathbf{M} \vee \mathbf{S}, \mathbf{M} \vee \mathbf{K}_{\mathbf{i}}(i=1,2,3), \mathbf{K}_{1} \vee \mathbf{K}_{2}, \mathbf{K}_{2} \vee \mathbf{K}_{3}, \mathbf{M} \vee \mathbf{K}_{1} \vee \mathbf{S}, \mathbf{M} \vee \mathbf{K}_{1} \vee \mathbf{K}_{2}, \mathbf{M} \vee \mathbf{K}_{2} \vee \mathbf{K}_{3}$. In order to pave the way for the statement of two theorems, which are tailor-made for our needs and due to $\mathrm{B}$. Davey and $\mathrm{H}$. Werner [8], we first present a definition.

Let $\mathbf{K}$ be a class of algebras and, for each $A \in \mathbf{K}$, let $\theta_{\mathbf{A}}(a, b)$ denote the principal congruence of $A$ collapsing a pair $a, b \in A$. Then a simplicity formula for $\mathbf{K}$ is a $\exists \forall$ conjunct of equations

$$
\sigma(u, v)=(\exists x)(\forall y)\left\{\sum_{i=1}^{n} p_{i}(x, y, u, v)=q_{i}(x, y, u, v)\right\}
$$

such that, for each $A \in \mathbf{K}, \sigma(u, v)$ holds in $A \Leftrightarrow \theta(u, v) \in\{\omega, \iota\}$.

Now we can state the theorems from Davey and Werner [7].

THEOREM 4. Let $\mathbf{K}$ be a congruence distributive variety generated by finitely many finite algebras. If there is a simplicity formula for the maximal subdirectly irreducible algebras in $\mathbf{K}$, then the injectives in $\mathbf{K}$ are precisely those algebras of the form $\prod_{j=1}^{n} A_{j}\left[B_{i}\right]$, where $\left\{A_{j} ; 1 \leqslant j \leqslant n\right\}$ is the set of all subdirectly irreducible algebras in $\mathbf{K}$ which are injective in $\mathbf{K}$ and $B_{j}$ is a complete Boolean algebra for each $j, 1 \leqslant j \leqslant n$.

The above theorem in conjunction with the next makes achievement of our goal a practicality.

THEOREM 5. Let $\mathbf{K}=\mathbf{S P}(\mathbf{A})$ for some finite set $\mathbf{A}$ of finite algebras. If $\mathbf{K}$ is congruence distributive and every member of $\mathbf{A}$ is either subdirectly irreducible or weak injective in $\mathbf{K}$, then $\mathbf{A} \in \mathbf{A}$ is injective in $\mathbf{K}$ if and only if it is injective in $\mathbf{A}$.

Obviously, every member $\mathbf{V}$ of $\Lambda \mathbf{M S}$ is congruence distributive, and, by the subdirect product theorem, $\mathbf{V}=\mathbf{S P}(\mathbf{S i}(\mathbf{V}))$. Since Goldberg [9] has shown that there is a simplicity formula for the class of all subdirectly irreducibles in $\mathbf{0}$, Theorems 4 and 5 tell us that the injectives in each (join reducible) member $\mathbf{V}$ of $\Lambda \mathbf{M S}$ are completely determined once the injectives in $\mathbf{S i}(\mathbf{V})$ are known.

Let $X, Y \in \mathbf{S i}(\mathbf{M S})$. Subsequently, when describing subalgebras of $X$ and extensions to $X$ of homomorphisms from them into $Y$, we shall use the same labelling of the elements of $X$ and $Y$ as that on the corresponding Hasse diagrams of $X$ and $Y$ which may be found in [2] and [4]. We will also make use of another consequence of Jónsson's lemma, namely that $\mathbf{S i}(\mathbf{X} \vee \mathbf{Y})=\mathbf{S i}(\mathbf{X}) \cup \mathbf{S i}(\mathbf{Y})$ and so $\mathbf{S i}(\mathbf{X} \vee \mathbf{Y})=\mathbf{S}(\{X\}) \cup \mathbf{S}(\{\mathbf{Y}\})$, by Lemma 2 .

Lemma 6. $K$ is the only non-trivial injective in $\mathbf{S i}(\mathbf{K} \vee \mathbf{S})$.

Proof. A glance at Fig. 2 confirms that $\mathbf{S i}(\mathbf{K} \vee \mathbf{S})=\{T, B, S, K\}$. Now observe that neither $B$ nor $S$ is injective in $\mathbf{S i}(\mathbf{K} \vee \mathbf{S})$. Indeed, the homomorphism from the subalgebra $B$ of $K$ into either $B$ or $S$ cannot be extended to $K$, since homomorphisms must map fixed points of the unary operation ${ }^{\circ}$ to fixed points, and, while $K$ has a fixed point, $B$ and $S$ have none. To see that $K$ is injective in $\mathbf{S i}(\mathbf{K} \vee \mathbf{S})$ it is enough, by reference to Fig. 2 and 
the fact that $K$ is injective in $\mathbf{K}$, to show that any homomorphism from any subalgebra of $S$ into $K$ extends to $S$. But $S(\{S\})=\{T, B, S\}$ and the homomorphism from the subalgebra $B$ of $S$ into $S$ can be extended to $S$ by mapping $a$ to 1 .

\section{Lemma 7. $K$ and $K_{1}$ are the only non-trivial injectives in $\mathbf{S i}\left(\mathbf{K}_{1} \vee \mathbf{S}\right)$.}

Proof. Clearly, $\mathbf{S i}\left(\mathbf{K}_{1} \vee \mathbf{S}\right)=\left\{T, B, S, K, K_{1}\right\} . B$ and $S$ are not injective in $\mathbf{S i}\left(\mathbf{K}_{1} \vee \mathbf{S}\right)$, since they are not in $\mathbf{S i}(\mathbf{K} \vee \mathbf{S})$ and $\mathbf{S i}(\mathbf{K} \vee \mathbf{S}) \subset \mathbf{S i}\left(\mathbf{K}_{1} \vee \mathbf{S}\right)$. That $K$ is injective in $\mathbf{S i}\left(\mathbf{K}_{1} \vee \mathbf{S}\right)$ follows on referring to Fig. 2 and using the fact that $K$ is injective in both $\mathbf{K}_{1}$ and $\mathbf{S i}(\mathbf{K} \vee \mathbf{S})$. To see that $K_{1}$ is injective in $\mathbf{S i}\left(\mathbf{K}_{1} \vee \mathbf{S}\right)$ it is sufficient, on referring to Fig. 2 and using the fact that $K_{1}$ is injective in $K_{1}$, to show that any homomorphism from any subalgebra of $S$ into $K_{1}$ extends to $S$. But $\mathbf{S}(\{S\})=\{T, B, S\}$ and the homomorphism from the subalgebra $B$ of $S$ into $K_{1}$ can be extended to $S$ by mapping $a$ to 1 .

LEMMA 8. $M$ is the only non-trivial injective in $\mathbf{S i}(\mathbf{V})$ for any proper subvariety $\mathbf{V}$ of MS containing $M$.

Proof. To show that $M$ is injective in $\mathbf{S i}(\mathbf{M} \vee \mathbf{S})$ and $\mathbf{S i}\left(\mathbf{M} \vee \mathbf{K}_{i}\right)$, for $i=1,2,3$, it suffices to show that $M$ is injective in $\mathbf{S i}\left(\mathbf{M} \vee \mathbf{K}_{i}\right)$ for $i=2$ and 3 , since $\mathbf{S i}(\mathbf{M} \vee \mathbf{S})$ and $\mathbf{S i}\left(\mathbf{M} \vee \mathbf{K}_{1}\right)$ are contained in $\mathbf{S i}\left(\mathbf{M} \vee \mathbf{K}_{3}\right)$. If we consult Fig. 2 and recall that $M$ is injective in $\mathbf{M}$ we see that it is only necessary to show that any homomorphism from any subalgebra of $K_{i}$ into $M$ extends to $K_{i}$ for $i=2$ and 3. Observe that $\mathbf{S}\left(\left\{K_{2}\right\}\right)=\left\{T, B, S, K, K_{2}\right\}$. Clearly, the homomorphism from the subalgebra $B$ of $K_{2}$ into $M$ can be extended by mapping $a$ to $a$ and $b$ to 1 . There are precisely two homomorphisms into $M$ from the subalgebra $K$ of $K_{2}$, namely $0 \mapsto 0, a \mapsto a, 1 \mapsto 1$ and $0 \mapsto 0, a \mapsto b, 1 \mapsto 1$, both of which can be extended to $K_{2}$ by mapping $b$ to 1 . There is one homomorphism into $M$ from the subalgebra $S$ of $K_{2}$, namely $0 \mapsto 0, b \mapsto 1,1 \mapsto 1$, and this can be extended to $K_{2}$ by mapping $a$ to $a$. Thus, $M$ is injective in $\mathbf{S i}\left(\mathbf{M} \vee \mathbf{K}_{2}\right)$. Next, observe that $\mathbf{S}\left(\left\{K_{3}\right\}\right)=\left\{T, B, K, S, K_{1}, K_{3}\right\}$. The homomorphism into $M$ from the subalgebra $B$ of $K_{3}$ can be extended by mapping $a$ and $c$ to $a$ and $b$ to 1 . There are two homomorphisms into $M$ from the subalgebra $K$ of $K_{3}$, namely $0 \mapsto 0, a \mapsto a, 1 \mapsto 1$, which can be extended to $K_{3}$ by mapping $c$ to $a$ and $b$ to 1 , and $0 \mapsto 0, a \mapsto b, 1 \mapsto 1$, which can be extended to $K_{3}$ by sending $c$ to $b$ and $b$ to 1 . There is only one homomorphism into $M$ from the subalgebra $S$ of $K_{3}$, namely $0 \mapsto 0, b \mapsto 1$, $1 \mapsto 1$, and this can be extended to $K_{3}$ by sending $a$ and $c$ to $a$. Finally, there are two homomorphisms into $M$ from the subalgebra $K_{1}$ of $K_{3}$, namely $0 \mapsto 0, a \mapsto a, c \mapsto a, 1 \mapsto 1$ and $0 \mapsto 0, a \mapsto b, c \mapsto b, 1 \mapsto 1$, both of which can be extended by mapping $b$ to 1 . Thus, $M$ is injective in $\mathbf{S i}\left(\mathbf{M} \vee \mathbf{K}_{3}\right)$.

In order to show that $M$ is the only non-trivial injective in $\mathbf{S i}(\mathbf{M} \vee \mathbf{S})$ and $\mathbf{S i}\left(\mathbf{M} \vee \mathbf{K}_{i}\right)$ for $i=1,2,3$, first observe that if $L \in\left\{K, K_{1}, K_{2}, K_{3}\right\}$ and $L \in \mathscr{A} \supseteq \mathbf{S i}(\mathbf{M})$, where $\mathscr{A} \subseteq$ $\operatorname{Si}(\mathbf{M S})$, then $L$ is not injective in $\mathscr{A}$. Indeed, any extension $h$ of the homomorphism from the subalgebra $B$ of $M$ into $L$ must satisfy $h(a)=h(b)=a$ so that $1=h(1)=h(a \vee b)=$ $h(a) \vee h(b)=a$, which is absurd. Now, $\operatorname{Si}(\mathbf{M} \vee \mathbf{S})=\{T, B, S, K, M\}, K$ is not injective in $\mathbf{S i}(\mathbf{M} \vee \mathbf{S})$ and $B$ and $S$ fail to be injective in $\mathbf{S i}(\mathbf{M} \vee \mathbf{S})$, since they fail to be injective in $\mathbf{S i}(\mathbf{K} \vee \mathbf{S})$. Therefore, $M$ is the only non-trivial injective in $\mathbf{S i}(\mathbf{M} \vee \mathbf{S})$. Observe that $\mathbf{S i}\left(\mathbf{M} \vee \mathbf{K}_{1}\right)=\left\{T, B, K, K_{1}, M\right\}, \quad \mathbf{S i}\left(\mathbf{M} \vee \mathbf{K}_{2}\right)=\left\{T, B, K, M, S, K_{2}\right\} \quad$ and $\quad \mathbf{S i}\left(\mathbf{M} \vee \mathbf{K}_{3}\right)=$ 
$\left\{T, B, K, M, S, K_{1}, K_{3}\right\} . K$ and $K_{1}$ are not injective in $\mathbf{S i}\left(\mathbf{M} \vee \mathbf{K}_{1}\right)$ and $B$ is not, because the homomorphism from the subalgebra $B$ of $K$ into $B$ obviously has no extension to $K$. $K$ and $K_{2}$ are not injective in $\operatorname{Si}\left(\mathbf{M} \vee K_{2}\right)$ and none of $K, K_{1}, K_{3}$ is injective in $\operatorname{Si}\left(\mathbf{M} \vee K_{3}\right)$. In addition, $B$ and $S$ are not injective in $\mathbf{S i}\left(\mathbf{M} \vee \mathbf{K}_{i}\right)$ for $i=2$ and 3 , since they are not in $\mathbf{S i}(\mathbf{M} \vee \mathbf{S})$. Thus, $\boldsymbol{M}$ is the only non-trivial injective in $\mathbf{S i}\left(\mathbf{M} \vee \mathbf{K}_{i}\right)$, for $i=1,2,3$. It is now straightforward to see that $M$ is the only non-trivial injective in $\mathbf{S i}\left(\mathbf{M} \vee K_{1} \vee \mathbf{S}\right), \mathbf{S i}\left(\mathbf{M} \vee K_{1} \vee\right.$ $\left.\mathbf{K}_{3}\right)$ and $\mathbf{S i}\left(\mathbf{M} \vee \mathbf{K}_{2} \vee \mathbf{K}_{3}\right)$.

Lemma 9. $K, K_{1}, K_{2}$ are the only non-trivial injectives in $\mathbf{S i}\left(\mathbf{K}_{1} \vee \mathbf{K}_{2}\right)$.

Proof. Note that $\operatorname{Si}\left(\mathbf{K}_{1} \vee \mathbf{K}_{2}\right)=\left\{T, B, K, S, K_{1}, K_{2}\right\}$ and that $B$ and $S$ are not injective in $\mathbf{S i}\left(\mathbf{K}_{1} \vee \mathbf{K}_{2}\right)$, since they are not injective in $\mathbf{S i}(\mathbf{K} \vee \mathbf{S}) \subset \mathbf{S i}\left(\mathbf{K}_{1} \vee \mathbf{K}_{2}\right)$. Referring to Fig. 2 and recalling that $K$ is injective in both $\mathbf{K}_{1}$ and $\mathbf{K}_{2}$, we see that $K$ is injective in $\mathrm{Si}\left(\mathbf{K}_{1} \vee \mathbf{K}_{2}\right)$. To show that $K_{1}$ is injective in $\operatorname{Si}\left(\mathbf{K}_{1} \vee \mathbf{K}_{2}\right)$ it is enough, since $K_{1}$ is injective in $\mathbf{K}_{1}$, to show that any homomorphism into $K_{1}$ from any member of $\mathbf{S}\left(\left\{K_{2}\right\}\right)$ extends to $K_{2}$. Clearly, the homomorphism into $K_{1}$ from the subalgebra $B$ of $K_{2}$ can be extended by mapping $a$ to $a$ and $b$ to 1 . There is exactly one homomorphism into $K_{1}$ from the subalgebra $K$ of $K_{2}$, namely $0 \mapsto 0, a \mapsto a, 1 \mapsto 1$, which can be extended to $K_{2}$ by mapping $b$ to 1 , and there is exactly one homomorphism into $K_{1}$ from the subalgebra $S$ of $K_{2}$, namely $0 \mapsto 0, b \mapsto 1,1 \mapsto 1$, which can be extended to $K_{2}$ by mapping $a$ to $a$. It remains to show that $K_{2}$ is injective in $\mathbf{S i}\left(\mathbf{K}_{1} \vee \mathbf{K}_{2}\right)$. Since $K_{2}$ is injective in $\mathbf{K}_{2}$, it is enough to show that any homomorphism into $K_{2}$ from any member of $\mathbf{S}\left(\left\{K_{1}\right\}\right)$ can be extended to $K_{1}$. Clearly, the homomorphism into $K_{2}$ from the subalgebra $B$ of $K_{1}$ can be extended by mapping both $a$ and $b$ to $a$. There is exactly one homomorphism into $K_{2}$ from the subalgebra $K$ of $K_{1}$, namely $0 \mapsto 0, a \mapsto a, 1 \mapsto 1$, and this can be extended by mapping $b$ to $a$.

Lemma 10. $K$ is the only non-trivial injective in $\mathbf{S i}\left(\mathbf{K}_{2} \vee \mathbf{K}_{3}\right)$.

Proof. Observe that $\operatorname{Si}\left(\mathbf{K}_{2} \vee \mathbf{K}_{3}\right)=\left\{T, B, K, S, K_{1}, K_{2}, K_{3}\right\}$ and that neither $B$ nor $S$ is injective in $\mathbf{S i}\left(\mathbf{K}_{2} \vee \mathbf{K}_{3}\right)$, since they are not injective in $\mathbf{S i}\left(\mathbf{K}_{1} \vee \mathbf{K}_{2}\right) . K_{1}$ is not injective in $\mathrm{Si}\left(\mathbf{K}_{2} \vee \mathbf{K}_{3}\right)$ because the homomorphism into $K_{1}$ from the subalgebra $K_{1}$ of $K_{3}$ given by $0 \mapsto 0, a \mapsto a, c \mapsto b, 1 \mapsto 1$ cannot be extended to $K_{3}$. Indeed, any such extension $h$ must satisfy $1=h(1)=h(a \vee b)=h(a) \vee h(b)=a \vee h(b)$, so that $h(b)=1$, and then $b=h(c)=$ $h(a \wedge b)=h(a) \wedge h(b)=a$, which is absurd. That $K_{2}$ fails to be injective in $\mathbf{S i}\left(\mathbf{K}_{2} \vee \mathbf{K}_{3}\right)$ follows from the observation that the homomorphism into $K_{2}$ from the subalgebra $S$ of $K_{3}$ given by $0 \mapsto 0, b \mapsto b, 1 \mapsto 1$ has no extension to $K_{3}$; any such extension $h$ must satisfy $h(a)=a$ so that $1=h(1)=h(a \vee b)=h(a) \vee h(b)=a \vee b=b . K_{3}$ also fails to be injective in $\mathbf{S i}\left(\mathbf{K}_{2} \vee \mathbf{K}_{3}\right)$ because the homomorphism into $K_{3}$ from the subalgebra $S$ of $K_{2}$ given by $0 \mapsto 0, b \mapsto b, 1 \mapsto 1$ does not extend to $K_{2}$; any such extension $h$ must satisfy $h(a)=a$ so that $b=h(b)=h(a \vee b)=h(a) \vee h(b)=a \vee b=1$. Finally, $K$ is injective in $\mathbf{S i}\left(\mathbf{K}_{2} \vee \mathbf{K}_{3}\right)$, since it is injective in both $\mathbf{K}_{2}$ and $\mathbf{K}_{3}$.

In order to be economical in summarizing our results, we adopt the following notation. $\mathscr{B}$ will stand for the class of all complete Boolean algebras. If $\mathbf{V} \in \Lambda \mathbf{M S}$ then $\operatorname{Inj}(\mathbf{V})$ will denote the class of all injective algebras in $\mathbf{V}$. 
THEOREM 11. The injective algebras in each of the twenty subvarieties of the variety MS are as follows:

(1) $\operatorname{Inj}(\mathbf{T})=\{\mathbf{T}\}$

(2) $\operatorname{Inj}(\mathbf{B})=\mathscr{B}$,

(3) $\operatorname{Inj}(\mathbf{S})=\left\{B_{0} \times S\left[B_{1}\right] ; B_{0}, B_{1} \in \mathscr{B}\right\}$,

(4) $\operatorname{Inj}(\mathbf{K})=\operatorname{Inj}(\mathbf{K} \vee S)=\operatorname{Inj}\left(\mathbf{K}_{2} \vee \mathbf{K}_{3}\right)=\left\{K\left[B_{0}\right] ; B_{0} \in \mathscr{B}\right\}$,

(5) $\operatorname{Inj}\left(\mathbf{K}_{1}\right)=\operatorname{Inj}\left(\mathbf{K}_{1} \vee \mathbf{S}\right)=\left\{K\left[B_{0}\right] \times K_{1}\left[B_{1}\right] ; B_{0}, B_{1} \in \mathscr{B}\right\}$,

(6) $\operatorname{Inj}\left(\mathbf{K}_{2}\right)=\left\{K\left[B_{0}\right] \times K_{2}\left[B_{1}\right] ; B_{0}, B_{1} \in \mathscr{B}\right\}$,

(7) $\operatorname{Inj}\left(\mathbf{K}_{3}\right)=\left\{K\left[B_{0}\right] \times K_{3}\left[B_{1}\right] ; B_{0}, B_{1} \in \mathscr{B}\right\}$,

(8) $\operatorname{Inj}\left(\mathbf{M}_{1}\right)=\left\{M\left[B_{0}\right] \times M_{1}\left[B_{1}\right] ; B_{0}, B_{1} \in \mathscr{B}\right\}$,

(9) $\operatorname{Inj}\left(\mathbf{K}_{1} \vee \mathbf{K}_{2}\right)=\left\{K\left[B_{0}\right] \times K_{1}\left[B_{1}\right] \times K_{2}\left[B_{2}\right] ; B_{0}, B_{1}, B_{2} \in \mathscr{B}\right\}$,

(10) if $\mathbf{V}$ is a proper subvariety of $\mathbf{M S}$ containing $\mathbf{M}$ then $\operatorname{Inj}(\mathbf{V})=\left\{M\left[B_{0}\right], B_{0} \in \mathscr{B}\right\}$.

COROLlary 12. All join irreducibles in $\Lambda \mathbf{M S}$ have enough injectives but $\mathbf{K}_{1} \vee \mathbf{K}_{2}$ is the only join reducible in $\Lambda \mathrm{MS}$ having enough injectives.

The injectives in all subvarieties of $\mathbf{M}$ have been described already in the literature (see [9] and the references therein). An equivalent structure theorem for the injectives in $\mathbf{S}$, the variety of Stone algebras, was first obtained by $\mathbf{R}$. Balbes and G. Grätzer (see [1] or [10] and the references therein).

\section{REFERENCES}

1. R. Balbes and P. Dwinger, Distributive lattices, (University of Missouri Press, 1974). appear).

2. R. Beazer, On some small varieties of distributive Ockham algebras, Glasgow Math. J. (to

3. J. Berman, Distributive lattices with an additional unary operation, Aequationes Math. 16 (1977), 165-171.

4. T. S. Blyth and J. C. Varlet, On a common abstraction of de Morgan algebras and Stone algebras, Proc. Roy. Soc. Edinburgh Sect. A 94A (1983), 301-308.

5. T. S. Blyth and J. C. Varlet, Subvarieties of the class of MS-algebras, Proc. Roy. Soc. Edinburgh Sect. A 95A (1983), 157-169.

6. S. Burris and H. P. Sankappanavar, A course in universal algebra, (Springer-Verlag, 1981).

7. B. Davey, On the lattice of subvarieties, Houston J. Math. 5 (1979), 183-192.

8. B. Davey and H. Werner, Injectivity and Boolean powers, Math. Z. 166 (1979), 205-223.

9. M. S. Goldberg, Distributive Ockham algebras: free algebras and injectivity, Bull. Austral. Math. Soc. 24 (1981), 161-203.

10. G. Grätzer, General lattice theory, (Birkhauser Verlag, 1978).

11. H. A. Priestley, Representation of distributive lattices by means of ordered Stone spaces, Bull. London Math. Soc. 2 (1970), 186-190.

12. H. A. Priestley, Ordered topological spaces and the representation of distributive lattices, Proc. London Math. Soc. (3) 24, (1972), 507-530.

13. A. Urquhart, Distributive lattices with a dual homomorphic operation, Studia Logica 38 (1979), 201-209.

Department of Mathematics, UNIVERSITY OF GLASGOW, SCOTLAND. 